\title{
Feasibility and performance of lymphoscintigraphy in sentinel lymph node biopsy for early cervical cancer: results of the prospective multicenter SENTICOL study
}

\author{
Anne-Sophie Bats - Albane Frati - Marc Froissart - Isabelle Orliaguet • \\ Denis Querleu • Slimane Zerdoud • Eric Leblanc • Hélène Gauthier • \\ Catherine Uzan · Désirée Deandreis · Emile Darai · Khaldoun Kerrou • \\ Henri Marret · Emilie Lenain · Patrice Mathevet · Fabrice Lecuru
}

Received: 28 May 2014/ Accepted: 18 September 2014/Published online: 15 November 2014

(C) The Japanese Society of Nuclear Medicine 2014

\begin{abstract}
Objective To evaluate feasibility, SLN detection rate, and SLN location of lymphoscintigraphy in sentinel lymph node (SLN) biopsy for early cervical cancer.

Methods Ancillary analysis of data from the multicenter prospective SENTICOL study (January 2005-June 2007) of patients with early cervical cancer (FIGO stage IA with emboli to IB1) was conducted. Preoperative lymphoscintigraphy was performed after intracervical administration of 60 or $120 \mathrm{MBq}$ of ${ }^{99 \mathrm{~m}} \mathrm{Tc}$-labeled radiocolloid on the day before (long protocol) or morning of (short protocol) surgery. SLNs were identified intraoperatively using
\end{abstract}

A.-S. Bats and A. Frati contributed equally to this work and should be considered co-first authors.

A.-S. Bats - F. Lecuru

Faculté de Médecine, Université Paris Descartes, Sorbonne Paris Cité, Paris, France

\section{A.-S. Bats $(\varangle) \cdot$ A. Frati · F. Lecuru}

Chirurgie Cancérologique Gynécologique et du Sein, Hôpital Européen Georges-Pompidou, Assistance Publique-Hôpitaux de Paris, 20-40 rue Leblanc, 75015, Paris, France

e-mail: anne-sophie.bats@egp.aphp.fr

\section{A.-S. Bats $\cdot$ F. Lecuru}

INSERM UMR-S 1124, Université Paris Descartes, Sorbonne

Paris Cité, Paris, France

M. Froissart

Service de Médecine Nucléaire, Hôpital Européen Georges-

Pompidou, Assistance Publique-Hôpitaux de Paris, Paris, France

I. Orliaguet

Médecine Nucléaire, Hôpital Edouard Herriot, Lyon, France

D. Querleu

Chirurgie Générale, Institut Claudius Regaud, Toulouse, France combined radioactivity/patent blue detection. SLNs were sampled electively and routine bilateral pelvic lymphadenectomy was performed by laparoscopy. A centralized review of lymphoscintigraphies was performed to assess feasibility, detection rates, and anatomic SLN location. Results Of 139 patients included in the SENTICOL study, 133 received radiocolloid injection, and 131 (98.5\%) underwent preoperative lymphoscintigraphy, with the long protocol in three-fourths of cases. The lymphoscintigraphic detection rate was $87.8 \%$, with a median of 2 (1-4) SLNs per patient. By multivariate analysis, factors independently associated with lymphoscintigraphic SLN detection were age [odds ratio (OR) $0.91,95 \%$ confidence interval (95\% CI) 0.87-0.96; $P<0.001$ ] , and protocol (long vs. short; OR 8.23, $95 \%$ CI 1.87-36.25;

S. Zerdoud

Médecine Nucléaire, Institut Claudius Regaud, Toulouse, France

E. Leblanc

Chirurgie Générale, Center Oscar Lambret, Lille, France

H. Gauthier

Médecine Nucléaire, Center Oscar Lambret, Lille, France

C. Uzan

Chirurgie Générale, Institut Gustave Roussy, Villejuif, France

D. Deandreis

Médecine Nucléaire, Institut Gustave Roussy, Villejuif, France

E. Darai

Gynécologie-Obstétrique, Hôpital Tenon, Assistance Publique-

Hôpitaux de Paris, Paris, France

K. Kerrou

Médecine Nucléaire, Hôpital Tenon, Assistance Publique-

Hôpitaux de Paris, Paris, France 
$P=0.005)$. Bilateral SLN identification by lymphoscintigraphy occurred in $67 \%$ of cases and was independently influenced by age (OR $0.95,95 \%$ CI $0.92-0.98$, $P<0.001)$ and protocol (OR 5.42, $95 \%$ CI 2.21-13.27; $P<0.001)$. Although $60.5 \%$ of preoperative SLNs were in the external iliac territory, unusual drainage patterns included the common iliac (19.6\%), para-aortic (10.8\%), and parametrial $(6 \%)$ basins.

Conclusions Our study demonstrates the feasibility and good detection rate of preoperative lymphoscintigraphy, with better detection in younger patients and with the long protocol. The high proportion of SLN basins in unexpected territories is of interest to guide intraoperative detection. Further studies are needed to better evaluate preoperative detection and to assess the contribution of lymphoscintigraphy to intraoperative detection.

Keywords Sentinel lymph node - Cervical cancer . Lymphoscintigraphy $\cdot$ Feasibility $\cdot$ Detection

\section{Introduction}

The sentinel lymph node (SLN) biopsy procedure was developed to identify and analyze the first drainage nodes of solid tumors, whose status is representative of the nodes located further along the drainage pathway. A negative SLN biopsy indicates that complete lymphadenectomy is unnecessary, thus allowing a substantial decrease in surgical morbidity. In addition, the SLN technique, and particularly preoperative lymphoscintigraphy, may help to detect aberrant drainage pathways and allows nodal ultrastaging, which is difficult to perform on a large number of nodes.

Among gynecological cancers, early cervical cancer seems a good candidate for the SLN technique. Nodal involvement is a major prognostic factor in early cervical cancer [1], but is present in less than $20 \%$ of patients at this stage [2]. Nodal recurrences have been reported

H. Marret

Gynécologie-Obstétrique, Hôpital Bretonneau, CHRU de Tours, Tours, France

E. Lenain

Département d'Informatique Hospitalière, Evaluation et Gestion des Connaissances, Hôpital Européen Georges-Pompidou,

Assistance Publique-Hôpitaux de Paris, Paris, France

P. Mathevet

Chirurgie Gynécologique, Hôpital Femme-Mère-Enfant, Bron, France

P. Mathevet

Université Lyon I, Lyon, France outside the usually sampled territories [3], and most of the involved nodes correspond to low-volume disease [4]. Extensive studies of SLN biopsy in this indication [5-7] have established feasibility and shown excellent detection rates and diagnostic value [8].

The SLN technique initially relied on the injection of patent blue dye. Subsequently, radiocolloid injection followed by preoperative lymphoscintigraphy and intraoperative gamma-probe detection was found to increase the SLN detection rate and the sensitivity of the technique [9, 10]. The impact of the intraoperative detection method (patent blue, radiocolloid, or both) on the detection and diagnostic value of SLN biopsy has been extensively studied. In contrast, few data are available on SLN detection by preoperative lymphoscintigraphy. We recently reported [8] that lymphoscintigraphy reduced the risk of false-negative SLN detection in patients with early cervical cancer.

The purpose of this study was to evaluate the feasibility, SLN detection rate, factors influencing the detection, and SLNs anatomic location of lymphoscintigraphy in patients with early cervical cancer. To achieve these objectives, we analyzed data from the large prospective multicenter SENTICOL study $[8,11]$.

\section{Materials and methods}

\section{Materials}

This work is an ancillary analysis of data from the prospective multicenter SENTICOL study performed between January 2005 and June 2007 in seven French tertiary centers with expertise in laparoscopic SLN biopsy to assess the usefulness of this procedure in patients with early cervical cancer. Inclusion criteria of the SENTICOL study were squamous cell carcinoma, adenocarcinoma, or adenosquamous carcinoma of the cervix FIGO stage IA1 with emboli to IB1; the diagnosis was confirmed by biopsy or conization, clinical examination, and pelvic MRI. Noninclusion criteria were downstaging after chemotherapy or radiotherapy, documented allergy to patent blue or rhenium sulfide, medical history of severe allergy, age less than 18 years, pregnancy, and preoperative imaging findings suggesting lymph node involvement. Written informed consent was obtained from each patient before study inclusion, and the study was approved by the appropriate ethics committee (Comité de Protection des Personnes HEGP-Broussais, Paris). This study included 139 patients in the modified intention-to-diagnose population [8]. Table 1 reports the main characteristics of the patients and tumors. The results of intraoperative SLN detection have been reported $[8,11]$. 
Table 1 Characteristics of the study patients and tumors

\begin{tabular}{lc}
\hline Characteristics & $n=139(\%)$ \\
\hline Mean age $( \pm \mathrm{SD})$, years & $44.4( \pm 13.6)$ \\
Mean BMI $( \pm \mathrm{SD}), \mathrm{kg} / \mathrm{m}^{2}$ & $23.7( \pm 5.5)$ \\
Past history of cone biopsy, $n(\%)$ & $62(44.6)$ \\
Histology, $n(\%)$ & \\
Squamous cell carcinoma & $103(74.1)$ \\
Adenocarcinoma & $34(24.4)$ \\
Adenosquamous carcinoma, $n$ & $2(1.4)$ \\
FIGO stage, $n(\%)$ & \\
IA1 with emboli & $5(3.6)$ \\
IA2 & $12(8.6)$ \\
IB1 & $121(87.1)$ \\
IIA & $1(0.7)$ \\
\hline
\end{tabular}

$B M I$ body mass index, FIGO federation of gynecology and obstetrics

\section{Methods}

A $4 \mathrm{~mL}$-radiocolloid (99mTc-labeled colloidal rhenium sulfide, Nanocis ${ }^{\circledR}$, Cis Bio International, Gif-sur-Yvette, France) injection into the four cardinal points of the cervix was performed using a 25 -gauge needle on the day before (120 MBq, long protocol) or morning of $(60 \mathrm{MBq}$, short protocol) surgery. A lymphoscintigraphy was performed the day before or the day of surgery depending on the centres, and early and delayed scintigraphic planar images with anterior and lateral views were obtained. The results were communicated to the surgeons before the procedure (Fig. 1a, b).

At the beginning of the surgical procedure, $2 \mathrm{~mL}$ of diluted patent blue ( $2.5 \%$ in $2 \mathrm{~mL}$ of saline) was injected into the four cardinal points of the cervix. Intraoperative detection was performed before and after opening the peritoneum, pararectal, and paravesical spaces. The SLNs were detected based on the blue color of the lymphatic channels and nodes and on radioactivity detected using a handheld gamma probe. SLNs were electively sampled. The level of radioactivity in each SLN was measured in vivo and ex vivo, and the absence of residual in vivo radioactivity was checked. Pelvic lymphadenectomy was then performed routinely. Depending on the center, frozensection histology was performed either routinely or only on nodes with gross changes suggesting metastasis. In the absence of node metastasis, colpohysterectomy was performed. Patients with nodal involvement underwent pelvic and para-aortic lymphadenectomy, but not hysterectomy, as chemoradiotherapy was indicated.

The numbers of SLNs detected pre-and intraoperatively were recorded, as well as their anatomic location according to Marnitz et al. [12]. Typical drainage was defined as through the external iliac and interiliac basins. All other

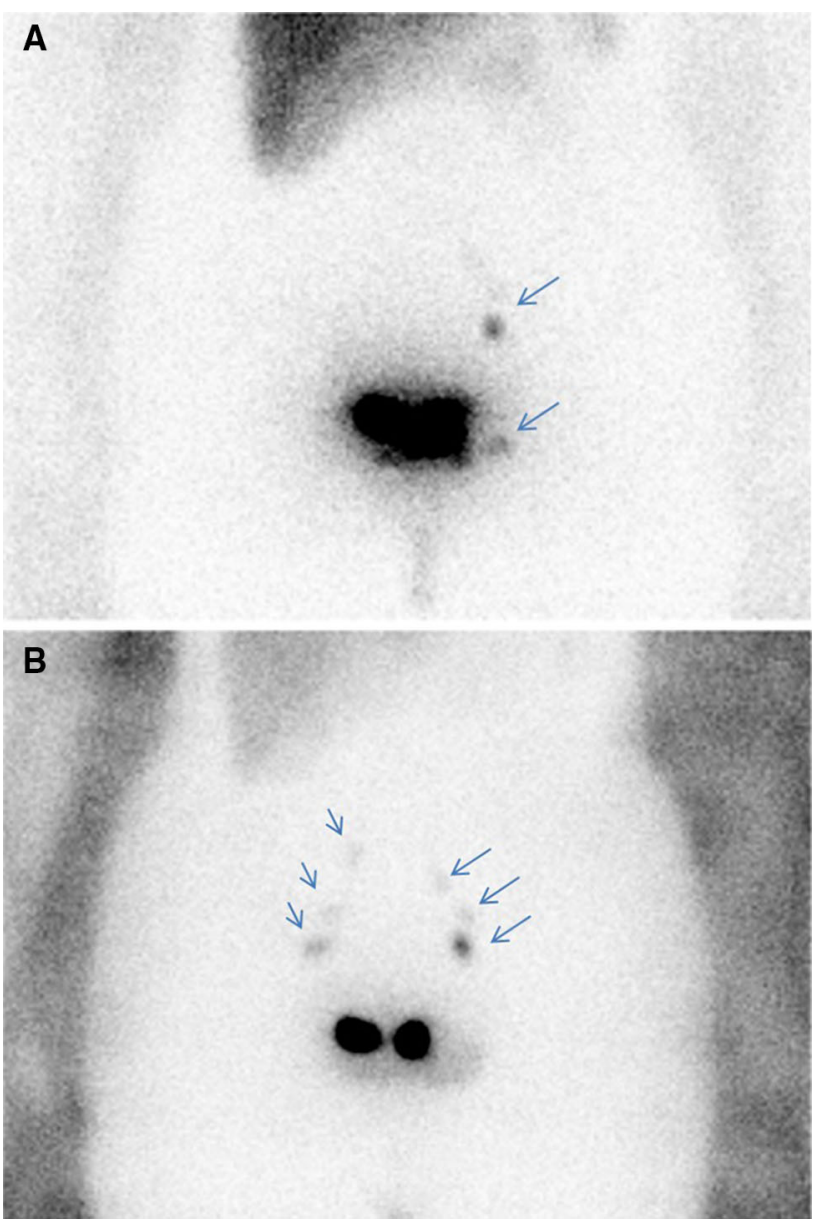

Fig. 1 Preoperative sentinel lymph node (SLN) detection in a patient with early cervical cancer. a Planar image $30 \mathrm{~min}$ after injection of Nanocis ${ }^{\circledR}(4 \times 400 \mu \mathrm{Ci})$ : identification of two SLNs on the left side, one in the inguinal territory and the other in the iliac territory. b Planar image 90 min after injection of Nanocis ${ }^{\circledR}(4 \times 400 \mu \mathrm{Ci})$ : identification of three SLNs in the left iliac territory and four SLNs in the right iliac territory

drainage basins were considered atypical. Histological examination of all SLNs was performed after hematoxylineosin-saffron (HES) staining of $200-\mu$ sections. SLNs negative by HES were examined by immunohistochemistry with anti-cytokeratin AE1-AE3 (Dako, France). Non-SLNs were examined routinely after HES staining at the local center, by the pathologist who examined the SLNs. All pathologists who participated in the study were trained in SLN biopsy for breast, cervical, and endometrial cancers. Isolated tumor cells were defined as $<0.2 \mathrm{~mm}$, micrometastases as between 0.2 and $2 \mathrm{~mm}$, and macrometastases as $>2 \mathrm{~mm}$ [13].

For the analysis of lymphoscintigraphic detection, all lymphoscintigrams were subjected to a centralized review by two experienced nuclear physicians and three surgeons (MF, IO, FL, PM, ASB). All radioactive LNs were considered as SLNs. Based on the results, we 
Table 2 Preoperative detection of sentinel lymph nodes using the short and long protocols
Q1-Q3 interquartile range from the 25th to the 75th percentile

SLN sentinel lymph node, NS nonsignificant

\begin{tabular}{|c|c|c|c|c|}
\hline & Total & $\begin{array}{l}\text { Short protocol } \\
n=31\end{array}$ & $\begin{array}{l}\text { Long protocol } \\
n=98\end{array}$ & $P$ value \\
\hline $\begin{array}{l}\text { Lymphoscintigraphic detection } \\
\text { rate, } n(\%)\end{array}$ & $100 / 114(87.8)$ & $20 / 27(74.1)$ & 79/86 (91.9) & 0.020 \\
\hline $\begin{array}{l}\text { Bilateral lymphoscintigraphic } \\
\text { detection rate, } n(\%)\end{array}$ & $67 / 114(58.8)$ & $14 / 27$ (51.9) & $52 / 86(60.5)$ & NS \\
\hline $\begin{array}{l}\text { Median number of SLNs per } \\
\text { patient, (Q1-Q3) }\end{array}$ & $2(1-4)$ & $2(0-3)$ & $2(1-4)$ & NS \\
\hline $\begin{array}{l}\text { Median number of right-sided } \\
\text { SLNs per patient (Q1-Q3) }\end{array}$ & $1(0-2)$ & $1(0-1)$ & $1(0-2)$ & NS \\
\hline $\begin{array}{l}\text { Median number of left-sided SLNs } \\
\text { per patient (Q1-Q3) }\end{array}$ & $1(0-2)$ & $1(0-1)$ & $1(1-2)$ & NS \\
\hline $\begin{array}{l}\text { Median time from radiotracer } \\
\text { injection to lymphoscintigraphy, } \\
\text { minutes (Q1-Q3) }\end{array}$ & $146(35-900)$ & $61(45-82)$ & $840(30-900)$ & $<0.001$ \\
\hline $\begin{array}{l}\text { Median time from } \\
\text { lymphoscintigraphy to surgery, } \\
\text { min (Q1-Q3) }\end{array}$ & $180(112-1156)$ & $99(64-242)$ & $293(135-1246)$ & $<0.001$ \\
\hline
\end{tabular}

\section{Results}

determined the lymphoscintigraphic detection rate and the factors affecting it, the bilateral detection rate, and the anatomic location of SLNs detected by lymphoscintigraphy.

\section{Statistical analysis}

As previously described [8], the sample size $(n=120$ patients) was calculated assuming a $20 \%$ prevalence of lymph node involvement (1), a $90 \%$ detection rate, a sensitivity per patient of $87.5 \%$, a sensitivity of $63-97 \%$, and a negative predictive value of 90-99\% [14]. The study was carried out using the modified intention-to-diagnose approach. We first determined the detection rate. The feasibility of preoperative lymphoscintigraphy was assessed by collecting failures and complications. Pain during the injections was evaluated by the patients on a visual analog scale (VAS). The times from injection to lymphoscintigraphy and from lymphoscintigraphy to surgery were recorded.

We investigated the potential impact on lymphoscintigraphic detection of age, history of conization, body mass index (BMI), histological type, presence of emboli, and protocol (long or short). For the univariate analysis, we used logistic regression models to identify predictors of any SLN detection and of bilateral SLN detection. $P$ values lower than 0.05 were considered significant. Variables from univariate analysis yielding $P$ values lower than 0.20 were included in a multivariate logistic model associated with the dependent variable [15], in which $P$ values lower than 0.05 were considered significant. All analyses were performed using SAS software (release 9.2; SAS Institute, Cary, NC, USA).
Sentinel lymph node (SLN) technique and feasibility of lymphoscintigraphy

The radiocolloid injection was performed in 133 patients, of whom $131(98.5 \%)$ underwent preoperative lymphoscintigraphy; in the remaining 2 patients, technical problems precluded lymphoscintigraphy. The short protocol was used in $31(23.7 \%)$ patients and the long protocol in $98(74.8 \%)$ patients; the protocol was not recorded for 2 patients. Table 2 shows the times from radiocolloid injection to lymphoscintigraphy and from lymphoscintigraphy to surgery. Among the 98 patients who had the radiocolloid injection on the day before surgery (long protocol), 42 underwent lymphoscintigraphy on the day before surgery and 47 on the day of surgery; this information was missing for 9 patients.

Only $1(0.8 \%)$ patient experienced a serious complication. The radiocolloid started to be injected, whereas she was at the beginning of a pregnancy. The injection was then stopped, but the pregnancy was terminated a few weeks later. No specific complications of lymphoscintigraphy were recorded. Pain during the injection was assessed in 46 of 131 patients: the VAS score was $<3$ in 29 $(63 \%)$ patients $[9(75 \%)$ receiving the short and 20 $(58.8 \%)$ the long protocol], 3-7 in $16(34.8 \%)$ patients [2 $(16.7 \%)$ receiving the short and $14(41.2 \%)$ the long protocol], and $>7$ in $1(2.2 \%)$ patient (short protocol). Of the 131 lymphoscintigraphies, 114 underwent centralized review.

All 139 patients in the modified intention-to-diagnose analysis received patent blue injection followed by SLN 
detection and biopsy then by pelvic and/or para-aortic lymphadenectomy.

Preoperative lymphoscintigraphic detection of sentinel lymph nodes (SLNs)

\section{Preoperative lymphoscintigraphic SLN detection rate}

The centralized review of 114 lymphoscintigrams identified 352 SLNs in 100/114 (87.8\%) patients. Median number of SLNs detected per patient was $2(1-4)$ overall, 1 $(0-2)$ for SLNs on the right side, and $1(0-2)$ for SLNs on the left side. SLNs were detected bilaterally in $67 / 100$ (67\%) patients. Table 2 reports the data separately for the short and long protocols.

Factors influencing preoperative lymphoscintigraphic SLN detection

By univariate analysis, factor significantly associated with a higher lymphoscintigraphic SLN detection rate was long protocol (OR 3.95, $95 \%$ CI 1.22-12.85, $P=0.022$ ). Older age was associated with a lower detection rate (OR 0.51 , $95 \%$ CI $0.33-0.75, P<0.001)$.

For the multivariate analysis, we used the two factors above, BMI $(P=0.056)$, and time from radiocolloid injection to lymphoscintigraphy $(P=0.134)$. Factors independently associated with SLN detection in the multivariate model were older age (OR 0.91, $95 \%$ CI $0.87-0.96 ; P<0.001)$ and long protocol (OR 8.23, $95 \%$ CI 1.87-36.25, $P=0.005$ ). Higher BMI showed a trend toward a lower detection rate (OR 0.91, $95 \%$ CI $0.82-1.01, P=0.072$ ).

\section{Factors influencing bilateral preoperative lymphoscintigraphic SLN detection}

By univariate analysis, factors significantly influencing bilateral SLN detection were age (OR 0.95, $95 \%$ CI $0.93-0.98, P=0.003$ ) and time from radiocolloid injection to lymphoscintigraphy (OR 4.29, $95 \%$ CI 1.95-9.90, $P<0.001)$. Bilateral detection decreased with older age and increased with longer time from radiocolloid injection to lymphoscintigraphy.

The multivariate model was built using the two factors above $(P$ values $<0.20)$, BMI $(P=0.079)$, histological type $(P=0.104)$, and histological differentiation $(P=0.163)$. In this model, factors independently associated with bilateral SLN detection were age (OR 0.95, $95 \%$ CI $0.92-0.98, P<0.001)$ and time from radiocolloid injection to lymphoscintigraphy (OR 5.42, $95 \%$ CI 2.21-13.27, $P<0.001)$.
Table 3 Location of sentinel lymph nodes detected preoperatively by centralized review of lymphoscintigraphies and intraoperatively based on blue color and/or radioactivity

\begin{tabular}{lll}
\hline Location & $\begin{array}{l}\text { Preoperative SLNs, } \\
n(\%)(n=352)\end{array}$ & $\begin{array}{l}\text { Intraoperative SLNs, } \\
n(\%)(n=419)\end{array}$ \\
\hline $\begin{array}{l}\text { External iliac }+ \text { inter- } \\
\text { iliac + internal iliac }\end{array}$ & $213(60.41 \%)$ & $334(80.29 \%)$ \\
Common iliac & $69(19.6 \%)$ & $34(8.11 \%)$ \\
Inguinal & 0 & 0 \\
Para-aortic & $38(10.79 \%)$ & $15(3.58 \%)$ \\
Parametrium & $21(6 \%)$ & $30(7.16 \%)$ \\
Promontory & $10(2.8 \%)$ & $5(1.19 \%)$ \\
Mid-abdomen & 0 & 0 \\
Other (presacral) & $1(0.3 \%)$ & 0 \\
\hline
\end{tabular}

$S L N$ sentinel lymph node

\section{Location of SLNs detected by preoperative lymphoscintigraphy}

Most of the SLNs detected by lymphoscintigraphy were located in the external iliac territory $(60.5 \%)$, as expected. However, substantial percentages of SLNs were in the common iliac $(19.6 \%)$ and para-aortic $(10.8 \%)$ basins. The percentage of parametrial SLNs was $6 \%$ (Table 3 ).

Intraoperative detection of sentinel lymph nodes (SLNs)

Intraoperative detection was performed in 136 of the 139 patients. The intraoperative detection rate using combined patent blue and radiocolloid detection was $97.8 \%$ (89.9\% blue and $93.9 \%$ radioactive SLNs). In all, 419 SLNs were identified (61 blue, 109 radioactive, and 249 both). Median number of SLNs detected intraoperatively per patient was 3 (2-4) overall and 1 on each side $(1,2)$. SLNs were detected bilaterally in $104(76.5 \%)$ patients. Most of the SLNs detected intraoperatively were located in the external iliac basin $(79.7 \%$ ) but $8.1 \%$ were in the common iliac basin, $3.6 \%$ in the para-aortic basin, and $7.1 \%$ in the parametrium.

Histological results

Of 136 patients with detected SLNs, 23 had positive SLNs and 2 had negative SLNs with positive non-SLNs. In both patients with false-negative results, the metastatic lymph nodes were located in the interiliac basin and were evenly distributed between the left and right sides. In one of these patients, a single SLN was detected by lymphoscintigraphy and during surgery, on the right side, and the metastatic non-SLN was on the left side. In the other patient, 
lymphoscintigraphy showed six bilateral SLNs, whereas a single SLN was detected intraoperatively, based on blue color; none of the nodes were radioactive. The time from radiocolloid injection to surgery in this patient was $16 \mathrm{~h}$ (960 min), which was less than the median.

Of 23 patients, 4 had metastatic SLNs in unexpected basins and $3(3 / 23,13.0 \%)$ had node metastases located only in unexpected basins and identified only by SLN biopsy.

\section{Discussion}

This large prospective, multicenter study assessed lymphoscintigraphy for the preoperative detection of SLNs in patients with early cervical cancer. Lymphoscintigraphy proved both feasible and safe. When the lymphoscintigrams were subjected to centralized review, the SLN detection rate was $87.8 \%$, which was slightly lower than the intraoperative detection rate. Importantly, younger age and radiotracer injection on the day before surgery (long protocol) were associated with higher SLN detection rates by lymphoscintigraphy. Another finding of major clinical significance is that, although most SLNs were located in the expected drainage basins (external iliac and interiliac), a substantial proportion were in unusual basins (common iliac, $19.6 \%$; para-aortic, $11.8 \%$; and parametrium, $6 \%)$.

The intensity of pain due to intracervical radiocolloid injection was usually acceptable, with only 1 patient reporting a VAS score greater than 7 . The long protocol seemed associated with greater pain intensity than the short protocol. However, the volume injected was identical in the two protocols, and the difference in the radioactivity dose cannot explain the pain. Premedication of the patients on the day of surgery may explain the lower pain intensity with the short protocol. In addition, pain was assessed in only 46/131 patients, which may have induced bias.

Our lymphoscintigraphic detection rate $(87.8 \%)$ was within the previously reported range of 81.1-96\% [1619]. Variability in detection rates may be ascribable to differences in injected doses and timing of the injection and lymphoscintigraphy. Importantly, we identified two factors associated with a higher detection rate, namely, younger age and long protocol (120 MBq on the day before surgery instead of $60 \mathrm{MBq}$ on the morning of surgery). The known decline in lymphatic system function with advancing age may explain that younger patients more often had detectable SLNs. The long protocol involved a substantially longer time interval between the radiocolloid injection and imaging, which may have allowed the radiocolloid to travel over longer distances, thus helping to detect second- or third-tier nodes [16], and increasing the detection rate.
We are aware of a single previous study, in only 32 patients, involving identification of factors associated with the SLN detection rate in patients with early-stage cervical cancer [19]. Other studies assessed only the contribution of lymphoscintigraphy to intraoperative SLN detection. Our results indicate a particularly important role for lymphoscintigraphy in younger patients and support the use of the long protocol.

The detection rate was lower by lymphoscintigraphy than by intraoperative examination, in keeping with earlier data. Intraoperatively, in addition to the radiocolloid, patent blue contributes to identify SLNs. In addition, studies indicate that surgeons use the lymphoscintigraphy findings to guide their exploration, thus increasing the likelihood of identifying SLNs [9, 20].

The SLNs detected by lymphoscintigraphy were usually located in the external iliac territory, as expected. However, $10.8 \%$ were in the para-aortic basin, which can be challenging to explore intraoperatively. The identification of aberrantly located drainage pathways or SLNs is a major advantage of lymphoscintigraphy. In particular, lymphoscintigraphy may show SLNs in the supra-mesenteric region, which is not usually well explored, due to the interposition of the gastrointestinal tract. The large amount of radiocolloid near the cervix limits the usefulness of lymphoscintigraphy for detecting parametrial SLNs. Nevertheless, $6 \%$ of SLNs seen by lymphoscintigraphy in our study were in the parametrial territory, and the proportion of SLNs at this location was similar by lymphoscintigraphy and by intraoperative detection. Thus, when interpreted by experts, lymphoscintigraphy is of interest for evaluating the parametrial nodes. In our study, the external iliac, interiliac, and common iliac node groups could not be reliably separated and were therefore classified as external iliac. Conventional lymphoscintigraphy produces 2-dimensional images with only anterior and lateral views, which is not sufficient to separate the external iliac, interiliac, internal iliac, presacral, and common iliac nodes. Imaging by combined single-photon-emission computed tomography (SPECT) and computed tomography (CT) has been found accurate in detecting aberrant drainage pathways, including the common iliac pathway (20). Detection rates were not significantly different between lymphoscintigraphy and SPECT-CT in this study, but this last method was more accurate in determining the anatomic location of the SLNs compared to planar lymphoscintigraphy. Several other studies obtained similar results [21-24]. Nevertheless, the detection rates by planar lymphoscintigraphy were lower in these studies than in ours. In addition, SLNs were more often detected in the common iliac territory by lymphoscintigraphy than by intraoperative detection, a fact that may be ascribable to the review of the images by nuclear medicine physicians. 
Our study has several limitations. The intraoperative detection protocol was standardized but the preoperative lymphoscintigraphy protocol was not. The radiocolloid was injected either on the day before surgery (long protocol) or on the morning of surgery (short protocol). Variations also occurred in the time from radiocolloid injection to imaging. The image acquisition modalities were not standardized. Furthermore all radioactive preoperative LNs were considered as SLNs, regardless of location, time of appearance, and node basin group, leading to overestimate the number of SLNs in some cases. In addition that intraoperative detection was performed with knowledge of the lymphoscintigraphy findings may have biased the comparison of pre-and intraoperative detection rates, thereby contributing to explain the higher intraoperative detection rate. However, in clinical practice lymphoscintigraphy is intended to facilitate intraoperative SLN detection, and blinding the surgeons to the lymphoscintigraphy findings would not have been ethically acceptable.

\section{Conclusion}

Our study demonstrates the feasibility of preoperative lymphoscintigraphy for SLN detection in patients with early cervical cancer. The lymphoscintigraphy detection rate, although lower than the intraoperative detection rate, was sufficiently high to suggest a role for preoperative lymphoscintigraphy, particularly using the long protocol. Moreover, the substantial proportion of SLNs identified by lymphoscintigraphy in unexpected territories is of major clinical significance. Surgeons can adjust their exploration procedure according to the lymphoscintigraphy results, thus increasing the likelihood of identifying SLNs. Further studies are needed to better evaluate preoperative lymphoscintigraphy detection of SLNs in patients with early cervical cancer. More specifically, the optimal lymphoscintigraphy protocol that maximizes the pre- and intraoperative detection rates must be defined.

\footnotetext{
Acknowledgments We are grateful to Gilles Chatellier (clinical trial administrator), Florence Gillaizeau (statistician), Noël Lucas (clinical trial administrator), Dominique Mariolle (financial account manager), Isabelle Sauret (clinical trial coordinator), Jean-François Leforestier (data manager), and Sonia Branco (clinical research associate) from the Unité de Recherche Clinique of the Hôpital Européen Georges-Pompidou, a publicly funded teaching hospital in Paris, France (Assistance Publique, Hôpitaux de Paris, Hôpital Européen Georges-Pompidou, Unité d'Epidémiologie et de Recherche Clinique, Paris, France). We thank Marie-Aude Le Frère-Belda (pathologist, Hôpital Européen Georges-Pompidou, Paris); Laurent Magaud (clinical trial coordinator, Hôpital Edouard Herriot, Hospices Civils de Lyon, Lyon); Isabelle Farre (pathologist, Centre Oscar Lambret, Lille); Pierre Duvillard (pathologist, Institut Gustave Roussy, Villejuif); Annie Cortez (pathologist, Hôpital Tenon, Assistance Publique-Hôpitaux de Paris, Paris); and Flavie Arbion
}

(pathologist, Hôpital Bretonneau, CHRU de Tours, Tours). We are indebted to Antoinette Wolfe, MD, for assistance in preparing and reviewing the manuscript. This study was funded by the following four French public funding agencies: AOR \#03063 grant to the National Hospital Clinical Research Program, 2003; French National Cancer Institute (INCA), Boulogne Billancourt, France; Programme Hospitalier de Recherche Clinique (PHRC), and Soutien aux Technologies et Innovations Coûteuses (STIC).

Conflict of interest The authors have not disclosed any potential conflict of interest.

\section{References}

1. Benedetti-Panici P, Maneschi F, Scambia G, Greggi S, Cutillo G, D'Andrea $G$, et al. Lymphatic spread of cervical cancer: an anatomical and pathological study based on 225 radical hysterectomies with systematic pelvic and aortic lymphadenectomy. Gynecol Oncol. 1996;62(1):19-24.

2. Fuller AF Jr, Elliott N, Kosloff C, Lewis JL Jr. Lymph node metastases from carcinoma of the cervix, stages IB and IIA: implications for prognosis and treatment. Gynecol Oncol. 1982;13(2):165-74.

3. Reiffenstuhl G. On the localization of lymphogenic seeding of cervical cancer. Wien Med Wochenschr. 1962;112:751-2.

4. Gortzak-Uzan L, Jimenez W, Nofech-Mozes S, Ismiil N, Khalifa MA, Dubé V, et al. Sentinel lymph node biopsy vs. pelvic lymphadenectomy in early stage cervical cancer: is it time to change the gold standard? Gynecol Oncol. 2010;116(1):28-32.

5. Dargent D, Martin X, Mathevet P. Laparoscopic assessment of the sentinel lymph node in early stage cervical cancer. Gynecol Oncol. 2000;79(3):411-5.

6. Malur S, Krause N, Köhler C, Schneider A. Sentinel lymph node detection in patients with cervical cancer. Gynecol Oncol. 2001;80(2):254-7.

7. Verheijen RH, Pijpers R, van Diest PJ, Burger CW, Buist MR, Kenemans P. Sentinel node detection in cervical cancer. Obstet Gynecol. 2000;96(1):135-8.

8. Lécuru F, Mathevet P, Querleu D, Leblanc E, Morice P, Daraï E, et al. Bilateral negative sentinel nodes accurately predict absence of lymph node metastasis in early cervical cancer: results of the SENTICOL study. J Clin Oncol. 2011;29(13):1686-91.

9. Plante M, Renaud MC, Têtu B, Harel F, Roy M. Laparoscopic sentinel node mapping in early-stage cervical cancer. Gynecol Oncol. 2003;91(3):494-503.

10. Lambaudie E, Collinet P, Narducci F, Sonoda Y, Papageorgiou T, Carpentier P, et al. Laparoscopic identification of sentinel lymph nodes in early stage cervical cancer: prospective study using a combination of patent blue dye injection and technetium radiocolloid injection. Gynecol Oncol. 2003;89(1):84-7.

11. Bats AS, Mathevet P, Buenerd A, Orliaguet I, Mery E, Zerdoud $\mathrm{S}$, et al. The sentinel node technique detects unexpected drainage pathways and allows nodal ultrastaging in early cervical cancer: insights from the multicenter prospective SENTICOL study. Ann Surg Oncol. 2013;20(2):413-22.

12. Marnitz S, Köhler C, Bongardt S, Braig U, Hertel H, Schneider A. German Association of Gynecologic Oncologists (AGO). Topographic distribution of sentinel lymph nodes in patients with cervical cancer. Gynecol Oncol. 2006;103(1):35-44.

13. Hermanek P, Hutter RV, Sobin LH, Wittekind C. International Union Against Cancer. Classification of isolated tumor cells and micrometastasis. Cancer. 1999;86(12):2668-73.

14. Levenback C, Coleman RL, Burke TW, Lin WM, Erdman W, Deavers $M$, et al. Lymphatic mapping and sentinel node 
identification in patients with cervix cancer undergoing radical hysterectomy and pelvic lymphadenectomy. J Clin Oncol. 2002;20(3):688-93.

15. Lemeshow S, Hosmer DW Jr. Estimating odds ratios with categorically scaled covariates in multiple logistic regression analysis. Am J Epidemiol. 1984;119:147-51.

16. Bats AS, Lavoué V, Rouzier R, Coutant C, Kerrou K, Darai E. Limits of day-before lymphoscintigraphy to localize sentinel nodes in women with cervical cancer. Ann Surg Oncol. 2008;15:2173-9.

17. Vieira SC, Sousa RB, Tavares M, Silva JB, Abreu BA, Santos LG, et al. Preoperative pelvic lymphoscintigraphy is of limited usefulness for sentinel lymph node détection in cervical cancer. Eur J Obstet Gynecol Reprod Biol. 2009;145:96-9.

18. Frumovitz M, Coleman RL, Gayed IW, Ramirez PT, Wolf JK, Gershenson DM, et al. Usefulness of preoperative lymphoscintigraphy in patients who undergo radical hysterectomy and pelvic lymphadenectomy for cervical cancer. Am J Obstet Gynecol. 2006;194:1186-93.

19. Kara P, Ayhan A, Caner B, Gültekin M, Ugur O, Bozkurt MF, et al. Sentinel lymph node détection in early stage cervical cancer: a prospective study comparing preoperative lymphoscintigraphy, intraoperative gamma probe and blue dye. Ann Nucl Med. 2008;22:487-94.
20. Martínez A, Zerdoud S, Mery E, Bouissou E, Ferron G, Querleu D. Hybrid imaging by SPECT/CT for sentinel lymph node detection in patients with cancer of the uterine cervix. Gynecol Oncol. 2010;119:431-5.

21. Pandit-Taskar N, Gemignani ML, Lyall A, Larson SM, Barakat RR, AbuRustum NR. Single photon emission computed tomography SPECT-CT improves sentinel node detection and localization in cervical and uterine malignancy. Gynecol Oncol. 2010;117(1):59-64.

22. Díaz-Feijoo B, Pérez-Benavente MA, Cabrera-Diaz S, Gil-Moreno A, Roca I, Franco-Camps S, et al. Change in clinical management of sentinel lymph node location in early stage cervical cancer: the role of SPECT/CT. Gynecol Oncol. 2011;120(3):353-7.

23. Buda A, Elisei F, Arosio M, Dolci C, Signorelli M, Perego P, et al. Integration of hybrid single-photon emission computed tomography/computed tomography in the preoperative assessment of sentinel node in patients with cervical and endometrial cancer: our experience and literature review. Int $\mathrm{J}$ Gynecol Cancer. 2012;22(5):830-5.

24. Kraft O, Havel M. Detection of sentinel lymph nodes in gynecologic tumours by planar scintigraphy and SPECT/CT. Mol Imaging Radionucl Ther. 2012;21(2):47-55. 